\title{
Sphingidae (Lepidoptera) preserved at the Museo Argentino de Ciencias Naturales, Buenos Aires: Catalog of the types and checklist of Argentinean species
}

\author{
Joanna RODRÍGUEZ-RAMÍREZ
}

División Entomología, Museo Argentino de Ciencias Naturales “Bernardino Rivadavia”, Av. Ángel Gallardo 470, C1405DJR, Ciudad de Buenos Aires, Argentina, joa.rodriguez@outlook.com.ar

\begin{abstract}
Resumen: Sphingidae (Lepidoptera) conservados en el Museo Argentino de Ciencias Naturales, Buenos Aires: Catálogo de los tipos y lista de las especies argentinas. Se presenta un catálogo de los ejemplares tipo existentes en el Museo Argentino de Ciencias Naturales "Bernardino Rivadavia" y una lista de las especies de Sphingidae de la Argentina preservadas en el museo. Diez especies nominales, nueve de la Argentina y una de Perú, están representadas por 13 ejemplares tipo. La lista de especies se basa en el examen de 1724 ejemplares. Se aportan numerosos nuevos registros para la mayoría de las especies, expandiendo la distribución de 25 especies a nuevas provincias.
\end{abstract}

Palabras clave: Especímenes tipo, nuevos registros, Argentina, Perú, Sphingidae.

\begin{abstract}
A catalog of all type specimens preserved in the Museo Argentino de Ciencias Naturales "Bernardino Rivadavia," and a checklist of species of Argentinean Sphingidae kept in the museum, are presented. Ten nominal species, nine from Argentina and one from Perú, are represented by 13 type specimens. The checklist is based on the examination of 1724 specimens. Numerous new records for most species are offered, with expansion of the distribution of 25 species to new provinces.
\end{abstract}

Key words: Type specimens, new records, Argentina, Perú, Sphingidae.

\section{INTRODUCTION}

Collections of insects are essential as they provide information on the entomological fauna inhabiting a given region. Also, collections are the repository of types, which are widely used as they are the necessary reference specimens for species identification decisions. The collection of the Entomological Division at the Museo Argentino de Ciencias Naturales "Bernardino Rivadavia" (MACN), in Buenos Aires, is the oldest and one of the largest in Argentina, covering a wealth of specimens perfectly preserved.

Lepidoptera being one of the best represented orders in number of specimens in colections, are well studied, in particular the family Sphingidae. Worldwide, about 200 genera and around 1350 species of hawkmoths (Kitching \& Cadiou, 2000) are known, out of which 30 genera with 122 species are present in Argentina (Kitching \& Cadiou, 2000; Núñez Bustos, com pers.). Sphingidae are among the main pollinators of the tropical communities, because they are the sole pollinators of most night-blooming flowers (Haber \& Frankie, 1989).

In Argentina, the first study of Sphingidae was published in the "Description Physique de la Republique Argentine", volumes I and II, and Atlas, by Burmeister (1878, 1879), who mentioned a total of 30 species. Subsequent works include those by Köhler (1924), Schreiter (1926) and Orfila (1933). Later publications of Schreiber (1978), Valverde (1999), Pastrana (2004), Moré et al. (2005), Nuñez-Bustos (2008, 2009), Mattoni and Penco (2012) and Nuñez-Bustos \& Rodríguez-Ramírez (2014) provide more records, even though information about species distribution in many regions of Argentina is still limited (Beccacece et al., 2011).

This contribution presents the results of the sorting, classifying and cataloging of the Sphingidae of the MACN collection. The databasing of all the information contained in specimen labels, provided new data that expand the distribution of many hawkmoths, specifically from Argentina. 


\section{MATERIALS AND METHODS}

The revision and sorting of the collection of Sphingidae was done between October 2012 and March 2014. The collection was reorganized, putting together the species from several collections, which had been kept separately. The type specimens and all specimens belonging to Argentina were entered in the MACN database, registering all the data on the labels. For the sorting and classification of the specimens Moré et al. (2005) and Oehlke (2014) were followed. Several identifications were confirmed by Ezequiel NuñezBustos.

The type specimens are alphabetically ordered, indicating the original genus of the binomen between brackets. The citation of the species is followed, between quotation marks, by the original statement of the author indicating locality, number of specimens, and other information considered useful. The specimens actually housed in the Museum are then mentioned, copying and describing their labels. Each label is copied between quotation marks, and lines on the labels are separated by a bar (/ ); characteristics of the label are mentioned between brackets. MACN-En numbers correspond to the entries in the database. Notes are provided, mentioned the present classification of each species. Each type specimen is illustrated in figure 1. Size measurements are also shown.

In the checklist the material examined is ordered by provinces and their departments. Species distributions are listed according to the literature and the studied material. Provinces cited in italics are new records for the species. Notes follow when appropriate.

\section{RESULTS}

The Sphingidae deposited in the MACN collection are represented by 27 genera and 79 species, classified into three sub-families. The number of genera and species per sub-family is as follows: Smerinthinae, 3 genera and 4 species, Sphinginae, 7 genera and 25 species and Macroglossinae, 17 genera and 50 species.

The specimens in the collection are well preserved, including those dating from the nineteenth century. The oldest specimens are dated between 1880 and 1898 , but most of the material is dated between 1901 and 1980, with recent collections in 1993-1998 and 2014. The amount of Argentine material of Sphingidae in the museum is 1,724 specimens. The Museum has lacked Lepidoptera specialists since the 1940 's. The speci- mens were spread in separate donations or material brought in by several collectors. This contribution is the result of the reorganization and cataloging the collection.

The numerous new records of Sphingidae in the MACN collection can be summarized indicating those provinces to which the distribution is expanded. New province records are as follows:

Buenos Aires: Pachylia syces (Hübner).

Catamarca: Erinnyis oenotrus (Cramer).

Chaco: Agrius cingulata (Fabricius), Manduca sexta paphus (Cramer), Erinnyis crameri (Schaus), Erinnyis domingonis (Butler), Erinnyis ello (Linnaeus) and Xylophanes ter$s a$ (Linnaeus).

Córdoba: Eumorpha fasciatus (Sulzer).

Corrientes: Agrius cingulata Fabricius.

Entre Ríos: Erinnyis ello (Linnaeus) and Eumorpha vitis (Linnaeus).

Formosa: Agrius cingulata Fabricius and Erinnyis alope (Drury).

Jujuy: Neogene reevei (Druce), Eumorpha labruscae (Linnaeus) and Hyles lineata (Fabricius).

La Pampa: Neogene reevei (Druce).

La Rioja: Manduca armatipes (Rothschild \& Jordan).

Mendoza: Manduca jordani (Giacomelli), Neogene reevei (Druce) and Hyles annei (Guérin-Meneville).

Misiones: Lintneira justiciae (Walker), Manduca contracta (Butler), Neogene reevei (Druce), Erinnyis domingonis (Bluter) and Madoryx oiclus (Cramer).

Neuquén: Manduca sexta paphus (Cramer) and Erinnyis ello (Linnaeus).

Río Negro: Manduca sexta paphus (Cramer).

Salta: Euryglottis guttiventris (Rothschild \& Jordan) and Eumorpha fasciatus (Sulzer).

San Juan: Erinnyis ello (Linnaeus) and Hyles euphorbiarum (Guérin-Méneville \& Percheron).

San Luis: Neogene reevei (Druce), Erinnyis oenotrus (Cramer) and Hyles lineata Fabricius.

Santa Fe: Eumorpha fasciatus (Sulzer) and Eumorpha labruscae (Linnaeus).

Santiago del Estero: Neogene reevei (Druce).

Tucumán: Adhemarius daphne (Boisduval), Orecta lycidas eos (Burmeister) and Manduca lefeburii (Guérin-Méneville).

\section{Catalog of the types}

baruta [Sphinx] Berg, 1883: 151-152. "Patria: Buenos Aires. De esta especie ha recogido el Sr. Günther un ejemplar en los talleres del Ferrocarril del Oeste, que conserva en su colección."

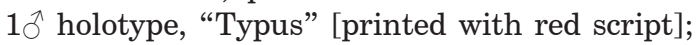
"Buen. Air. / col. Günther" [printed with red 
script]; "Neogene / reevei Druce / det. L. Valverde 1986" [manuscript]; "MACN-En 11692" [printed label]. Fig. 1a.

Note: Presently Neogene reevei (Druce, 1882).

bergi [Protoparce] Rothschild \& Jordan, 1903: 67, 94, 826. "Hab. Tucumán, Argentina. We name the species in memory of the late Professor Carlos Berg. In the Tring Museum 4 $\widehat{\jmath}, 1$ 우 from Tucumán; and $2 \widehat{0}$ from Argentina."

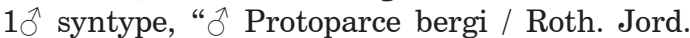
/ ..a..(illegible) Prestom (? unreadable) / Giac.

/ Tipo / Tucumán" [manuscript]; "MACN-En 11285" [printed label]. Fig. 1 b.

Note: Presently Manduca bergi (Rothschild \& Jordan, 1903). Given that maintained the exchange E. Giacomelli with K. Jordan, consider this specimen belongs to the type series.

cercyon [Dilophonota] Burmeister, 1878: 332333. "Not stated provenance, however indicates "Elle vit sur la même aspèce de Morrenia avec la chenille de D. Hippothoon". Dilophonota hippothoon is described in the same job with origin of Buenos Aires."

1 ㅇ syntype, "Cercyon / Burm / Buen. Air." [manuscript]; "Argentina / Buenos Aires / Mus. Nac. Hist. Natural Buenos Aires" [manuscript]; " 36 " [manuscript]; "HOLOTYPUS" [manuscript in red label]; "MACN-En 10921" [printed label]. Fig. 1c.

Note: Burmeister does not mention the number of specimens studied. Only one female remains. Presently Erinnyis lassauxii (Boisduval, 1859).

eos [Pilampelus] Burmeister, 1878: 350. "L`espèce fut dècouverte en 1865 à Buénos-Ayres par M. Kinkelin, ... qui a cédé au Musée le seul exemplaire d'une femelle prise entre Belgrano et Palermo ...; un second exemplaire male, un peu plus fort dessiné et coloré, je l'ai examiné dans la collection de M. Ruscheweyh, également pris à Buénos Ayres."

1 ^઼ syntype, "Lycidas / Bsd / eos Brm" [manuscript]; "Col. / Antigua" [printed label]; "Capital Federal / Buenos Aires / Argentina / 1865. Kinkelin" [manuscript]; "62" [manuscript]; "HOLOTYPUS" [manuscript in red label]; "envelope with pieces of legs"; "MACN-En 10918" [printed label]. Fig. 1d.

Note: Labels "Capital Federal / Buenos Aires / Argentina / 1865. Kinkelin" and "Holotypus" have handwriting of R. Orfila. The male specimen preserved is possibly the one collected by M. Ruscheweyh, whose collection was incorpo- rated into the museum. Presently Orecta lycidas eos (Burmeister, 1878).

hippothoon [Dilophonota] Burmeister, 1878: 333-334, Atlas pl. X, fig. 5. "L`espèce se trouve aux environs de Buénos-Ayres; nous l'ayons éduquée plusieurs fois de la chenille."

1 ex. syntype, "Buen. Ayres" [printed label in green paper]; "Col. / Antigua" [printed label]; "COTYPUS" [printed label in red cardboard]; "MACN-En 12280" [printed label]. Fig. 1e.

Note: Only one specimen was found, no determination label. Presently Erinnyis oenotrus (Cramer, 1780).

jordani [Protoparce] Giacomelli, 1912: 55-57. "Hábitat: República Argentina: Tucumán, La Rioja. “... Benjamín Martúret encontró un ejemplar en La Rioja, ejemplar desgastado; un año después, el naturalista doctor M. Lillo obtuvo otro de Tucumán ... que queda como "tipo". El ejemplar "co-tipo" desgastado ..., fue enviado a Tring (Inglaterra) al doctor K. Jordan que lo examinó y lo declaró como nueva especie".

$1 \uparrow$ holotype, “o??? Protoparce / jordani / Giac. n. sp. / (Año 1912) / Tipo - Tucumán” [manuscript]; "MACN-En 10925" [printed label]. Fig. 1f.

Note: Presently Manduca jordani (Giacomelli, 1912).

maura [Sphinx] Burmeister, 1879: 57-58. "Il a été envoyé de Tucuman à M. Kinkelin."

1ð syntype, "Tucumán / Argentina / Kinkelin leg. / Mus. Nac. Hist. Natural Buenos Aires" [manuscript]; "HOLOTYPO" [printed in red label]; "tipo / maura / Burm." [manuscript]; "28" [manuscript]; "Col. / Antigua" [printed label]; "HOLOTIPO" [printed in red label]; "MACN-En 10919" [printed label]. Fig. 1g.

Note: The single preserved specimen is considered a syntype because Burmeister did not indicate the number of studied specimens. Presently Lintneria maura (Burmeister, 1879).

panaquire [Sphinx] Berg, 1885: 266-267. "De esta especie fueron traídos varios ejemplares de Salta, por el Sr. Profesor Liberani, que se conservan en el Museo Público (MACN), en el Gabinete de Historia Natural de la Universidad (MACN) y en la colección del Sr. Ruscheweyh. Este último obtuvo también un ejemplar de Catamarca." $1{ }^{\Uparrow}$ syntype, "Salta" [manuscript]; "1" [manuscript]; a piece of leg mounted on white cardboard on the edges and transparent card; "Typus" [print with red script]; "Col. / Antigua" [printed 
label]; "17" [manuscript]; "Salta - Argentina / Berg. An. Soc. Cient. / Arg. XIX: 3-4 / R. \& J. Rev. Sphing.: / 75) / Mus. Nac. His. Natural Buenos Aires" [manuscript]; "COTYPUS" [manuscript in red label]; "MACN-En 10907" [printed label]. 1 ○ै syntype, "Tipo / Panaquire / Berg" [manuscript]; "17" [manuscript]; "Salta, Argentina / (An. Soc. Cient. Arg. / XIX: 3-4) / Mus. Nac. Hist. Natural Buenos Aires" [manuscript]; "Col. / Antigua" [printed label]; "COTYPUS" [manuscript in red label]; "MACN-En 10908" [printed label].

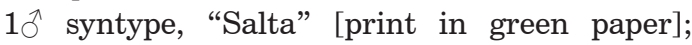
"TYPUS" [print in red label]; "MACN-En 11290" [printed label]. Fig. 1h.

Note: Presently Manduca contracta (Butler, 1875).

tucumana [Protoparce] Rothschild \& Jordan, 1903: 66, 81, 824. "Hab. Tucumán. $1{ }^{\uparrow}$ (type) in Mus. Tring; anoter of from Salta in the Museum at Buenos Ayres; a third from Paraguay in the Musée Royal d'Hist. Nat., Bruxelles; a fourth in the collection of Herr Neuburger, Berlin."

$1 \widehat{ }$ त paratype, "Salta" [manuscript in green paper]; "3" [manuscript]; genitalia mounted on white cardboard in the edges and transparent card; "Protoparce / tucumana / Cotypus 1902./ Nov. Zool." [manuscript]; "21" [manuscript]; "COTYPUS" [manuscript in red label]; "MACNEn 10922" [printed label]. Fig. 1i.

Note: Presently Manduca tucumana (Rothschild \& Jordan, 1903).

xylobotes [Choerocampa] (Burmeister, 1878): 355. "Notre collection possède deux exemplaires de cette espèce remarquable, communiqués par un ami de l'établissement, chassés par lui-même à Arica."

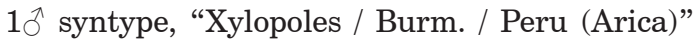
[manuscript]; "Col. / Antigua" [printed label]; "COTYPUS" [printed on red label]; "MACN-En 10923" [printed label].

10 syntype, "PERU / Arica" [manuscript]; "Col. / Antigua" [printed label]; "COTYPUS" [printed on red label]; "MACN-En 10924" [printed label]. Fig. 1j.

Note: Burmeister`s handwritten label says verbatim "Xylopoles". Presently Xylophanes xylobotes (Burmeister, 1878).

\section{Checklist of specimens}

\section{Smerinthinae \\ Ambulycini}

\section{Adhemarius daphne (Boisduval)}

Material examined: Misiones. Dpto. Calendaria: 11우, Loreto, Ogloblin col.;1우, Loreto, V-1936, Orfila col.; Dpto. Eldorado: 1ㅇ, Eldorado, 1-II-1943, Williner col.; Dpto. Frontera: $1{ }^{\lambda *}$, San Antonio, Viana col.; Dpto. Guarani: 1 ${ }^{*}$, El Soberbio, Viana col.; 1 ${ }^{\star}$, Montiagudo, Viana col.; Dpto. Iguazú: $4{ }^{\circ}, 5$ 우, A. Urugua-1́, IX-1954, Orfila col.; 1, XI-1910, Partridge col.; 2へ, 2ㅇ, XI-1954, Partridge col.; 2へ⿱ Iguazú, X-1928, Breyer col.; 1§̂, Iguazú, XII1933, Hayward col.; 1, Puerto Bemberg, 5-9-XII1933; đ, Puerto Cazador, Orfila col.; 19, Puerto Iguazú, 10-II-1944, Bridarolli col.; $1 \delta^{\Uparrow}$, Partridge col.; Dpto. San Pedro: 2 ${ }^{*}$, San Pedro, 1943, Viana col.; $1 \jmath^{\lambda}$, Orfila col.; Tucumán. Sin localidad: $1 \hat{\jmath}$, Breyer col.

Distribution: Misiones and Tucumán.

Note: Specimens marked with an asterisk are those cited as Adhemarius gannascus by Moré et al. (2005).

\section{Adhemarius gannascus (Stoll) \\ Material examined: Misiones. Dpto.}

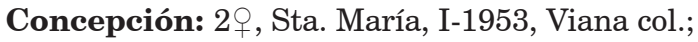
Dpto. Iguazú: 9 $\hat{\jmath}, 2$ ㅇ, A. Urugua-1́, IX-1954, Orfila col.; 10̂, Cataratas, X-1954, De Carlo \&Viana col.; 19, Puerto Iguazú, I-1944, Bridarolli col.; $2 \hat{\jmath}, 1$ 우, XI-1960, Partridge col.; $1 \hat{\jmath}$, 1 우, XI1954, Partridge col.; 3 ${ }^{2}$, Partridge col.; Sin localidad: $1 \widehat{ }{ }^{\lambda}$, Orfila col.; $1 \hat{\partial}$, Williner col.

Distribution: Corrientes, Misiones, and Salta.

\section{Orecta lycidas eos (Burmeister)}

Material examined: Buenos Aires. Type: $1 \delta$ syntype of Philampelus eos Burmeister (see above); Sin localidad: 1 , , 1880, Hilzinger col.; $1 \widehat{\jmath}, 1$, , Nosswitz col.; Misiones. Dpto. Iguazú: 1ð̂, Iguazú, I-1941, Louber col.; Tucumán.

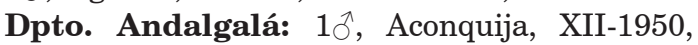
Golbach col.; Sin localidad: 19, 20-XI-1941, Hayward col.

Distribution: Buenos Aires, Corrientes, Misiones, and Tucumán.

Fig. 1. a. Sphinx baruta, holotype male (full length: 6,2 cm). b. Protoparce bergi, syntype male (full length: $9 \mathrm{~cm}$ ). c. Dilophonota cercyon, syntype female (full length: $10 \mathrm{~cm}$ ). d. Pilampelus eos, syntype male (full length: 7,9 cm). e. Dilophonota hippothoon, syntype (full length: 7,5 cm). f. Protoparce jordani, holotype female (full length: 7,2 cm). g. Sphinx maura, syntype male (full length: $9,5 \mathrm{~cm}$ ). h. Sphinx panaquire, syntype male (full length: $9,3 \mathrm{~cm}$ ). i. Protoparce tucumana, paratype male (full length: $9,3 \mathrm{~cm}$ ). j. Choerocampa xylobotes, syntype male (full length: $8 \mathrm{~cm}$ ). 


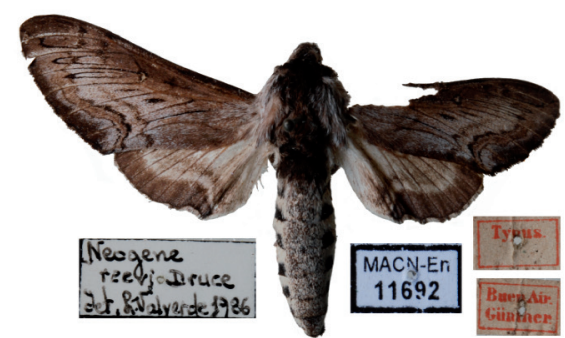

a
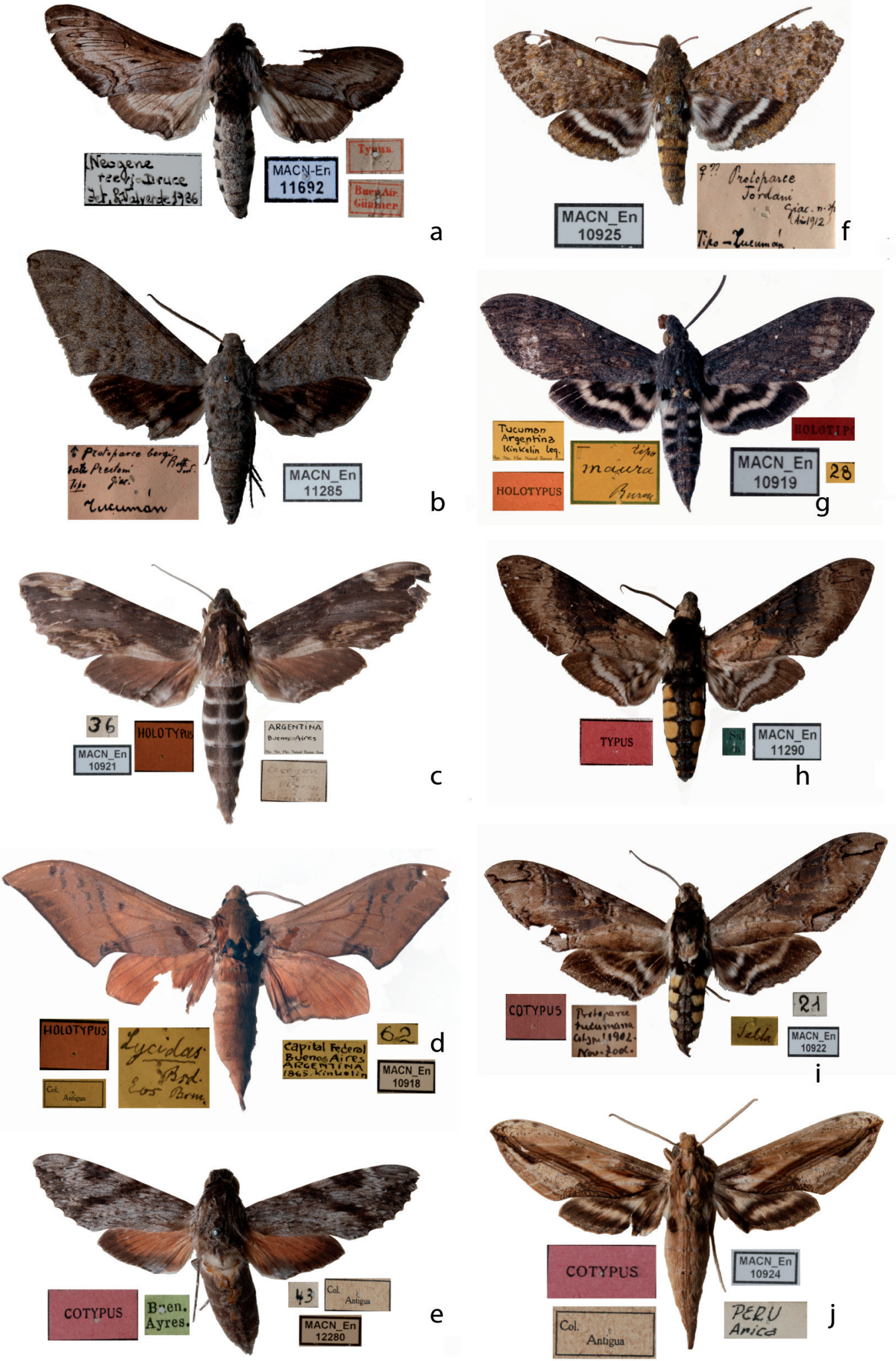


\section{Protambulyx strigilis (Linnaeus)}

Material examined: Misiones.

Calendaria: $1 \hat{\sigma}$, Loreto, V-1936; 1q, Loreto, Ogloblin col.; 1ð̂, Loreto; Dpto. Iguazú: 1, Puerto Bemberg, XII-1933, Hayward col.

Distribution: Formosa, Jujuy, Misiones, Salta, and Tucumán.

\section{Sphinginae}

Acherontiini

\section{Agrius cingulata Fabricius}

Material examined: Buenos Aires. Capital Federal: 1ðै, I-1929, Orfila col.; 19, III-1931, Orfila

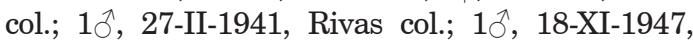

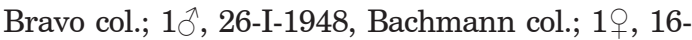
III-1944, Bachmann col.; 1ð̄, 5-9-XII-1933, Cando col.; Quilmes: 1ㅇ, 26-II-1936, Pérez-Moreau col.; Chaco. Sin localidad: 1 , Lynch col.; $1 \hat{O}, 22-$ IX-1915; Córdoba. Dpto. Calamuchita: 10, Los Reartes, 1972, Viana col.; 1ㅇ, La Tablada; Corrientes. Dpto. San Cosme: 1오 San Cosme, 11-V-1938; Formosa, Dpto. Formosa: 1 $\delta^{\lambda}$, Gran Guardia; Misiones. Dpto. Calendaria:

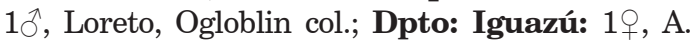
Urugua-1́, IX-1954, Orfila col.; Tucumán. Dpto.

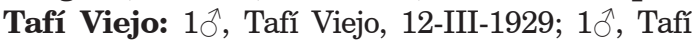
Viejo, X-1947, Orfila col.; 1옹 Manchalá, X-1922. Sin localidad: $2 \widehat{\jmath}, 2$,, $23-\mathrm{IX}-1919$.

Distribution: Buenos Aires, Chaco, Córdoba, Corrientes, Entre Ríos, Formosa, Jujuy, La Pampa, La Rioja, Mendoza, Misiones, Salta, San Juan, Santa Fe, and Tucumán.

\section{Sphinginae}

Sphingini

\section{Cocytius antaeus (Drury)}

Material examined: Misiones. Dpto. Candelaria: 1 , , Loreto, IV-1931; 10, camino Loreto-Santa Ana, Ogloblin col.; Dpto. Iguazú: 1우, Puerto Iguazú, 9-III-1934, Orfila col.; 1우, Puerto Iguazú, 9-III-1934, Hayward col.; Salta: $1 \widehat{\gamma}$, camino Tablillas-Vespucio, Harrington col.; Sin localidad: 1ิ̂, 20-I-1970, Köhler col.; Tucumán. Dpto. Tafí Viejo: $1 \hat{\delta}$, Tafí Viejo;

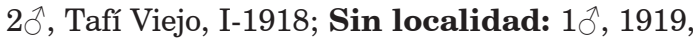
Schreiter col; 2 , , Nosswitz col.

Distribution: Buenos Aires, Chaco, Formosa, Misiones, Salta and Tucumán.

\section{Cocytius lucifer (Rothschild \& Jordan)} Material examined: Misiones. Dpto. Iguazú: 1오 Puerto Bemberg, 14-IV-1934, Hayward col. Distribution: Misiones.

\section{Euryglottis guttiventris (Rothschild \& Jordan)}

Material examined: Salta. Dpto. Capital: $1 \partial^{\lambda}$, Villa San Lorenzo, X-1959.

Distribution: Jujuy and Salta.

\section{Lintneria justiciae (Walker)}

Material examined: Misiones. Dpto. San Pedro: $1 \hat{\jmath}$, San Pedro, Viana col.

Distribution: Buenos Aires, Entre Ríos, and

Misiones.

\section{Lintneria maura (Burmeister)}

Material examined: Córdoba. Dpto. Calamuchita: $1 \hat{\jmath}$, Santa Rosa, 1972, Viana col.;

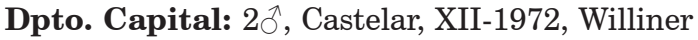
col.; ${ }^{2}, 1$, , La Tablada, Orfila col.; Dpto. Dpto. Lules: $1 \hat{\gamma}$, Quebrada de Lules, II-1928, Valverde col.; San Javier: 22 , La Paz, 1-15-I-1929, Bruch col.; Salta. Sin localidad: $1 \hat{\alpha}, 3-\mathrm{V}-1917$; Tucumán. Dpto. Chicligasta: 2^, Parque Nacional Campos Los Alisos, Puesto Santa Rosa, 14-III-2014, Rodríguez-Ramírez col.; 10ิ, Parque Nacional Campos Los Alisos, Puesto Los Chorizos, 15-16-III-2014, Rodríguez-Ramírez col.; Dpto. Tafí Viejo: $1 \hat{\partial}$, Tafí Viejo, II-1918; 1ㅇ, Tafí Viejo, 12-III-1929; 3今, Breyer col.; Type: $1{ }^{\lambda}$ syntype of Sphinx maura Burmeister (see above).

Distribution: Córdoba, Jujuy, La Rioja, Salta and Tucumán.

\section{Manduca albiplaga (Walker)}

Material examined: Misiones. Dpto. Iguazú:

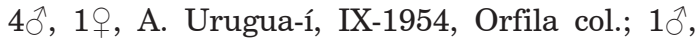
X-1928; 4ð, XI-1954, Partridge col.; Dpto. San Pedro: 10̂, San Pedro, 1943, Viana col.

Distribution: Jujuy and Misiones.

\section{Manduca armatipes (Rothschild \& Jordan)}

Material examined: Catamarca. Dpto.

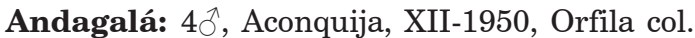
Salta. Dpto. Orán: 2 , Urundel, 26-III-1949,

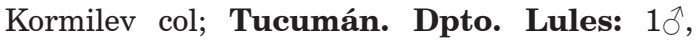
Quebrada de Lules, 30-XII-1926; Sin localidad: 1우, VII-1918, Reed col.; 1ðै, Schreiter col.

Distribution: Buenos Aires, Catamarca, Jujuy,

La Rioja, Salta and Tucumán.

\section{Manduca bergarmatipes (Clark)}

Material examined: Tucumán. Sin localidad:

$2 \widehat{\jmath}, 1$, , Schreiter col.

Distribution: Tucumán. 
Manduca bergi (Rothschild \& Jordan)

Material examined: Catamarca. Dpto. Capital:

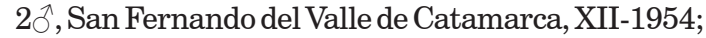

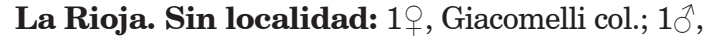
Orfila col.; Misiones. Dpto. Leandro N. Alem: $1 \delta$, Leandro N. Alem, 1952, Orfila col.; Tucumán. Dpto. Tafí Viejo: 1q, Tafí Viejo, I-1918; Dpto. Yerba Buena: $1 \hat{\jmath}$, Reserva Experimental Horco Molle, X-1960; Sin localidad: $6 \AA$, 1 우, Schreiter col.; Type: $1 \sigma^{\Uparrow}$ syntype de Protoparce bergi Rothschild \& Jordan (see above).

Distribution: Buenos Aires, Catamarca, Córdoba, Jujuy, La Rioja, Misiones, Salta and Tucumán.

\section{Manduca contracta (Butler)}

Material examined: Misiones. Dpto. Iguazú: 1 ${ }^{\lambda}$, Puerto Bemberg, XII-1933, Hayward col.; 1今, 24-X-1938, Hayward col.; Salta. Type: $2 \hat{\bigcirc}$ syntypes of Sphinx panaquire Berg (see above); Tucumán. Dpto. Tafí Viejo: $1 \widehat{\partial}$, Tafí Viejo, I-1918; 1ð̂, Manchalá, I-1922; 1ô, Manchalá, XII-1922.

Distribution: Buenos Aires, Corrientes, Entre Ríos, Misiones, Salta and Tucumán.

\section{Manduca diffissa diffissa (Butler)}

Material examined: Buenos Aires. Capital Federal: 1 $\hat{}$, I-1959, Partido General Pueyrredón: Orfila col.; 1언 Mar del Plata, 24-I-1927, Gramajo col.; Partido San Miguel:

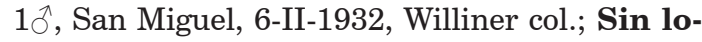
calidad: $1 \hat{\gamma}$, XII-1936, Orfila col.; $1 \hat{\jmath}, 1$, 1 , 2-IV1910. Córdoba. Dpto. Calamuchita: $1 \hat{\jmath}$, Los Reartes, 1972, Viana col.; Dpto. Capital: $1 \hat{\partial}$, Castelar, XI-1972, Williner col.; Dpto. Punilla: 1ิ̂, Los Cocos, 20-VIII-1916, Hauman col.; Sin

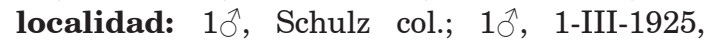
Brèthes col.

Distribution: Buenos Aires, Chaco, Córdoba and Entre Ríos.

\section{Manduca diffissa mesosa (Rothschild \& Jordan)}

Material examined: Catamarca. Dpto. Capital: 1 $\hat{O}^{\hat{2}}$, San Fernando del Valle de Catamarca, XI1940; Córdoba. Dpto. Calamuchita: $1 \widehat{\gamma}$, 1으, El Sauce, III-1972, Viana col.; Dpto. San Javier: 2 $\curvearrowright$, La Paz, 1-15-I-1929, Bruch col.; Dpto. Punilla: 1은 Los Cocos, 20-VIII-1916, Hauman col.; Sin localidad: 1 , Schulz col. San Juan. Dpto. Valle Fértil: 1ㅇ, 1, II-1972, Viana col.; Tucumán. Dpto. Andalagá: 1 $\widehat{\partial}$, Aconquija, XII-1950, Orfila col.; Dpto. Yerba Buena: 1으, Reserva Experimental Horco Molle, Orfila col.
Distribution: Catamarca, Córdoba, Jujuy, Mendoza, La Rioja, Salta, San Luis, San Juan and Tucumán.

\section{Manduca diffissa petuniae (Boisduval)}

Material examined: Misiones. Dpto. Iguazú: 10̂, A. Urugua-í, IX-1954, Orfila col.; Dpto. Leando N. Alem: 1 ${ }^{\hat{}}$, Leandro N. Alem, 1952, Orfila col.

Distribution: Misiones.

\section{Manduca florestan (Stoll)}

Material examined: Misiones. Dpto. Candelaria: 1 $\widehat{\partial}$, Loreto; Dpto. Iguazú: 1 $\widehat{\partial}$, A. Urugua-í, 20-26-VII-1957; 1ð̂, A. Urugua-í,

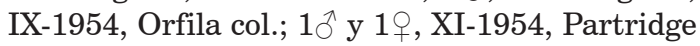
col.; $1{ }^{\lambda}$, Cataratas de Iguazú, X-1954, De Carlo \& Viana col.; Tucumán. Dpto. Lules: $1 \hat{\jmath}$, Quebrada de Lules, 30-XII-1926; 1ठ, Quebrada de Lules, II-1928; Dpto. Tafí Viejo: 1ㅇ, Tafí

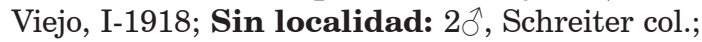
$1 \hat{\gamma}$, Bourquin col.; 2 ㅇ, Breyer col.

Distribution: Corrientes, Jujuy, La Rioja, Misiones, Salta, Santa Fe and Tucumán.

\section{Manduca jordani (Giacomelli)}

Material examined: Córdoba. Dpto. Punilla:

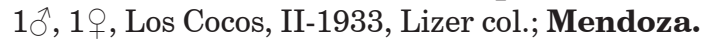
Dpto. Luján de Cuyo: 1으, Cacheuta. Tucumán. Type: 1 + Holotype (see above).

Distribution: Córdoba, La Rioja, Mendoza, Salta, San Luis and Tucumán.

\section{Manduca lefeburii (Guérin-Méneville)}

Material examined: Misiones. Dpto. Iguazú: 1ð̂, Roverano col.; Tucumán. Sin localidad: $1{ }^{\lambda}$, Valverde col.

Distribution: Misiones and Tucumán.

\section{Manduca lichenea (Burmeister)}

Material examined: Misiones. Dpto. Iguazú:

1ठ, XI-1964, Partridge col.

Distribution: Córdoba, Misiones, Salta and

Tucumán.

\section{Manduca rustica (Fabricius)}

Material examined: Buenos Aires. Capital Federal: 1 9, 27-II-1941, Rivas col.; Partido Almirante Brown: 1, Adrogué, 29-III-1909; 1 $\hat{\jmath}$, Adrogué; Partido Lomas de Zamora: 19, Lomas, III-1920; Partido San Isidro: 1ิึ, Martínez, XI-1926, Gómez col.; Partido Morón, 1ð̂, Castelar, XI-1965, Orfila col.; Sin localidad: 1 , IV-1929, Gallardo col.; 1ㅇ, 24III-1906, Brèthes col.; 10ิ, 10-I-1959, Moreno 
col.; 10, XII-1951, Orfila col.; 1우, 28-XII-1905; 3 , Bosq col.; Chaco. Dpto. Mayor Luis Jorge Fontana: 1今, Villa Angela, VI-1936, Castillo col.; Córdoba. Dpto. Ischilín: 10̂, Deán Funes; Dpto. Punilla: $1 \hat{\gamma}$, Capilla del Monte; $1 \hat{\gamma}, 1+$, Schulz col.; Corrientes. Sin localidad: $1 \hat{\jmath}$, 1935, Wurth col.; Misiones. Dpto. Apóstoles:

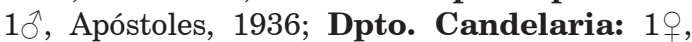
Loreto, IV-1951; 1ㅇ, Loreto, Bosq col.; Dpto. Iguazú: $2 \hat{\jmath}$, A. Urugua-í, IX-1954, Orfila col.; $6 \hat{\circ}$, Partridge col.; 1今, 1엉 XII-1933, Hayward col.; Dpto. San Pedro: $1 \AA$, San Pedro, 1943, Viana col.; Tucumán. Dpto. Tafí Viejo: $1 \hat{\jmath}, 1$, , Tafí Viejo, I-1918; Dpto. Chicligasta: 1 $\partial^{\lambda}$, Parque Nacional Campo Los Alisos, Puesto Santa Rosa, 14-III-2014, Rodríguez-Ramírez col.

Distribution: Buenos Aires, Chaco, Córdoba, Corrientes, Entre Ríos, Formosa, Jujuy, Misiones, Salta, Santiago del Estero, and Tucumán.

\section{Manduca sexta paphus (Cramer)}

Material examined: Buenos Aires. Capital federal: 1ㅇ, 11-IV-1930, Orfila col.; 10ิ, II-1949, Chabelski col.; Dpto. Saladillo: 1 오, Saladillo; 21-III-1998; Dpto. Tigre: 1 ${ }^{\wedge}, 3$, Tigre, II-1932, Bourquin col.; Partido de Coronel Suárez: $1 \hat{\jmath}$, Coronel Suárez, III-1985, Gallardo col.; Partido Pilar: 1, 1 $\delta^{\lambda}$, Zelaya, 19-II-1939, Chepliv col.; Partido San Isidro: 1ิे, Martínez, 13-IV-1916; Partido Tres Arroyos: 1 $\widehat{\jmath}$, Claromecó, II1954; 10, Sin localidad: 1-II-1937, Rodríguez col.; 1어, III-1938, Orfila col.; $1 \delta^{\jmath}$, I-1953, Orfila col.; 1ㅇ, 21-II-1910; 1ठ, 11-III-1910; 10, 18-III-

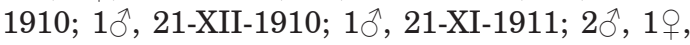
3-VII-1919; 1, III-1938, Orfila col.; Chaco. Dpto San Fernando: 1 $\hat{\jmath}$, Fontana, IV-1935, Meyer col.; Córdoba. Dpto. Calamuchita: $1 \hat{\jmath}$, Santa Rosa de Calamuchita, Viana col.; 2 , , Villa General Belgrano, 26-VIII-1971, Williner col.; 2ᄉ, 1ㅇ, El Sauce, III-1972, Viana col.; Dpto. Punilla: 2 $\hat{\circ}$, Capilla del Monte; Dpto. San

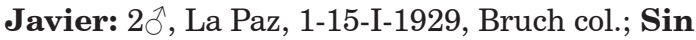
localidad: $1+$, Schulz col.; Corrientes. Dpto. San Cosme: $1 \hat{\jmath}$, San Cosme, Wurth col.; Sin localidad: $1 \hat{\jmath}, 1$, , II-1935, Bourquin col.; $1 \hat{\jmath}, 1$ 우, 1935, Wurth col.; Entre Ríos: $2 \widehat{\partial}$, Salto Grande, Obrador, 24-IV-1977, Williner col.; Jujuy: Dpto. Doctor Manuel Belgrano: 1 $\widehat{\jmath}$, San Salvador de Jujuy, 5-II-1949, De Martínez col.; La Pampa. Dpto. Chapaleufú: 1ð̂, Estación Sarah, 1-III1941, Benson col.; La Rioja: Sin localidad: 1 ${ }^{\gamma}$, II-1923; 1ㅇ, II-1929; Mendoza. Dpto. La Paz y Juan Martín de Pueyrredón: 1웅 Desaguadero, XII-1972, Williner col.; Misiones. Dpto Guarini: 1 $\widehat{\partial}$, El Soberbio, Viana col.;
Dpto. Iguazú: 1우, Partridge col.; 1ㅅ, XI-1950, Partridge col.; 1§̂, 1우 XII-1933, Hayward col.; 1ð̄, 4ㅇ, A. Urugua-1́, IX-1954, Orfila col.; Dpto. Leandro N. Alem: 1 $\hat{\jmath}$, 1q, Leandro N. Alem, 1952, Orfila col.; Neuquén. Dpto. Lácar: 1 $\widehat{\jmath}$, San Martín de los Andes, II-1953, Patrowsky col.; 1ठ․, Bajada del Marucho, 8-XII-1969, Schajovskoi col.; Sin localidad: $1 \jmath^{\hat{n}}$, 1ㅇ, 2-II-1952; Río Negro: Dpto. General Roca: 1 9 , Allen, I-1956, Quant col.; 10, Cipolletti, 21-II-1960, Schajovskoi col.; San Luis. Dpto. La Capital: 1ㅇ, San Gerónimo, II-1972, Viana col.; 19, San Gerónimo, XI-1972, Williner col.; Santiago de Estero: 1q, Cuesta del Río Salado; Tucumán. Dpto. Andalgalá: 1q, Aconquija, XII-1950, Orfila col.; Sin localidad: 1 , , 13-3-1998.

Distribution: Buenos Aires, Chaco, Córdoba, Corrientes, Entre Ríos, Formosa, Jujuy, La Pampa, La Rioja, Mendoza, Misiones, Neuquén, Río Negro, Salta, San Luis, Santiago del Estero, and Tucumán.

\section{Manduca tucumana (Rothschild \& Jordan)}

Material examined: Salta. Type: $1 \widehat{\jmath}$ syntype Protoparce tucumana Rothschild \& Jordan (see above); Tucumán. Dpto. Yerba Buena: $2 \hat{\jmath}$, 1, Reserva Experimental Horco Molle, X-1960, Williner col.; Dpto. Chicligasta: 1q, Parque Nacional Campos Los Alisos, Puesto Santa Rosa, 14-III-2014, Rodríguez-Ramírez col.; Sin localidad: $1 \hat{\partial}$, Bourquin col.; 1 , Schreiter col. Distribution: Catamarca, Jujuy, Salta, and Tucumán.

Note: It should be noted that among the specimens of Manduca tucumana (Rothschild \& Jordan, 1903), two specimens coming from the Misiones province of year 1953 collected by Ricardo N. Orfila were found. This is remarkable because this species has been found only in the northeast of the country, just in Catamarca, Jujuy, Salta, and Tucumán. They are presumed to be two specimens with altered labels.

\section{Manduca undata (Rothschild \& Jordan)}

Material examined: Córdoba. Dpto. Calamuchita: 1ð̂, El Sauce, III-1972, Viana col.; Dpto. Colón: 1ð̂, Agua de Oro, I-1940, De Carlo col.; Dpto. San Javier: 1 $\hat{\jmath}$, La Paz, 1-15I-1929, Bruch col.; 1 $\hat{\jmath}$, Yacanto; $1 \hat{\jmath}$, La Tablada; Sin localidad: $1 \hat{\gamma}$, 29-I-1891, Schulz col.; 1 , Schulz col.; La Rioja. Sin localidad: $3 \hat{\jmath}, 5$,

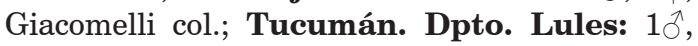
Quebrada de Lules, II-1928, Schreiter col.; Dpto. Yerba Buena: 1, Reserva Experimental Horco 
Molle, Orfila col.; Sin localidad: 10 , Schreiter col.;

Distribution: Córdoba, La Rioja, Salta, Santiago del Estero, and Tucumán

\section{Neococytius cluentius (Cramer)}

Material examined: Misiones. Dpto.

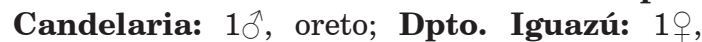
XI-1954, Partridge col.; 1ㅇ, Cataratas del Iguazú, 5-II-1934, Hayward col.; Santa Fe. Dpto Rosario: 1ㅇ, Rosario, 1932, Juillet col.; Tucumán: 1ㅇ, Manchalá, I-1920; 1, Manchalá, XII-1922.

Distribution: Chaco, La Rioja, Misiones, Salta, Santa Fe, and Tucumán.

\section{Neogene albescens Clark}

Material examined: Córdoba. Dpto. Calamuchita: $1 \hat{\alpha}$, El Sauce, III-1972, Viana col.; La Rioja. Sin localidad: $14 \hat{\jmath}, 3$, Giacomelli col.

Distribution: Córdoba, La Pampa, La Rioja, and Salta.

\section{Neogene reevei (Druce)}

Material examined: Buenos Aires. Partido Saladillo: $1 \hat{\partial}$, Saladillo, 23-III-1898; Sin localidad: $1 \hat{\partial}$, XI-1946, Ramos col.; Type: $1 \hat{\partial}$, Ciudad de Buenos Aires, Holotype of Sphinx baruta Berg (see above); Córdoba. Dpto. Calamuchita: $2 \widehat{\gamma}$, Santa Rosa de Calamuchita, XI-1972, Williner col.; $1 \hat{\partial}$, 3으, El Sauce, III-1972, Viana col.; Dpto. Capital: $1 \hat{\jmath}$, Castelar, II-1972, Williner col.; 2今, Castelar, XI-1972, Williner col.; Dpto. Colón: 1ㅇ, El Manzano, I-1942, Rodríguez col.; Dpto. Ischilín: 1엄 Deán Funes; Dpto. Santa María: 1ð, Anisacate, XII-1958, Hernández col.; Sin localidad: $1 \hat{\jmath}, 27-X I-1889$, Schulz col.;

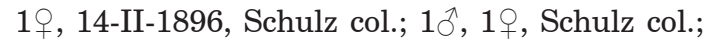
Jujuy. Dpto. Doctor Manuel Belgrano: 1 ${ }^{\lambda}$, San Salvador de Jujuy, XII-1949, Prosen col.; La Pampa. Dpto. Loventué: $1 \hat{\gamma}$, Luan Toro, 20-X-1953, Capdeville col.; La Rioja. Sin localidades: $6 \hat{\circ}, 7 \circ$, Giacomelli col.; Mendoza. Dpto. Santa Rosa: 1으, Reserva Nacuñan, 11-XI-1972, Williner col.; Misiones. Dpto. Candelaria: 1 ,,$\hat{\alpha}$, Loreto, Ogloblin col.; Salta. Dpto. General José de San Martín: 10̄, Río Carapari, 12-I-1945, Bridarolli col.; Dpto. Orán: 1우, El Tabacal, V-1933, Daguerre col.; San Luis. Dpto. La Capital: 1, San Gerónimo, 23-XI1971, Williner col.; 1오, San Gerónimo, XII-1972, Williner col.; Santiago del Estero: 1, Río Salado, Wagner col.

Distribution: Buenos Aires, Córdoba, Entre Ríos,
Jujuy, La Pampa, La Rioja, Mendoza, Misiones, Salta, San Luis, Santiago del Estero, Tucumán.

\section{Macroglossinae Dilophonotini}

\section{Aellopos clavipes (Rothschild \& Jordan)}

Material examined: Tucumán: 2?, Manchalá, III-1923.

Distribution: Salta and Tucumán.

\section{Aellopos tantalus (Linnaeus)}

Material examined: Buenos Aires. Partido Quilmes: 1ㅇ, Quilmes, 2-II-1919; Misiones.

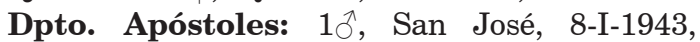

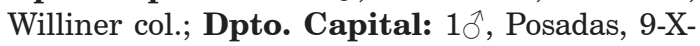
1933; Sin localidad: 1 , I-1943, Williner col.

Distribution: Buenos Aires, Catamarca, Chaco, Formosa, Misiones, Salta, and Tucumán.

\section{Aellopos titan titan (Cramer)}

Material examined: Buenos Aires. Capital Federal: 1우, Flores, 29-III-1919; 1ㅇ 8-I-1907, Brèthes col.; 1ð̂, II-1918; Misiones. Dpto. Capital: 1ㅇ, Posadas, 15-IX-1933; Dpto. Iguazú: 1ึ̂, A. Urugua-1́, IX-1954, Orfila col.; $2 \widehat{\gamma}$, XI-1950, Partridge col.; Salta. Dpto. Orán: 2 , , El Tabacal, I-1945, Williner col.

Distribution: Buenos Aires, Formosa, Misiones, Salta, and Tucumán.

\section{Aleuron iphis (Walker)}

Material examined: Misiones. Dpto. Apóstoles:

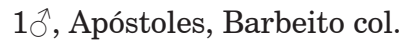

Distribution: Misiones.

\section{Aleuron neglectum (Rothschild \& Jordan)} Material examined: Misiones. Dpto.

Apóstoles: $2 \widehat{\jmath}$, Apóstoles, Barbeito col. Distribution: Misiones.

\section{Callionima grisescens grisescens} (Rothschild)

Material examined: Chaco. Dpto. Resistencia:

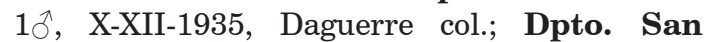
Fernando: 10̂, Fontana, IV-1935, Meyer col.; Córdoba. Dpto. La Paz: 1ㅇ, 1-15-I-1929, Bruch col.; 1 $\partial$, Sierras de Córdoba, 10-XI-1921, Williner col.; 1, La Tablada; Sin localidad: 1우 $1 \hat{\jmath}$, Schulz col.; Jujuy. Dpto Ledezma: 2ð, 28-XI-1978, Williner col.; La Rioja. Dpto. Independencia: $1 \hat{\jmath}$, Patquía, Breyer col.; Dpto. Independencia: 2 , , Patquía; 1 , , V-1914; $1 \widehat{\jmath}$, 2 , Giacomelli col.; Misiones. Dpto. Frontera: $3 \widehat{\partial}$, Piñalito, X-1954, De Carlo \& Viana col.; 
Dpto. Iguazú: 2ð, Cataratas de Iguazú, X-1954, De Carlo \& Viana col.; Salta. Dpto. General

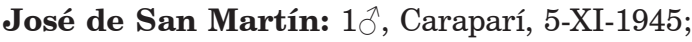
Santiago del Estero: 1 , Río Salado, Wagner col.; Tucumán. Dpto. Chiclígasta: $1 \widehat{\gamma}$, Parque Nacional Los Alisos, Puesto Los Chorizos, 1516-III-2014, Rodríguez-Ramírez col.; Dpto. Lules: 1 $\partial^{\lambda}$, Quebrada de Lules, XI-1922; $2 \hat{\circ}, 1$, Quebrada de Lules, II-1928; 1+, 1897; Dpto. Tafí Viejo: 1ð̂, Tafí Viejo, X-1947, Orfila col.; Dpto. Yerba Buena: $3 \hat{\circ}, 6 \circ$, Reserva Experimental Horco Molle, X-1960, Williner col.; Sin localidad: $1 \hat{\gamma}, 1$, , Schreiter col.

Distribution: Catamarca, Chaco, Córdoba, Jujuy, La Rioja, Misiones, Salta, San Luis, Santiago del Estero, and Tucumán.

\section{Callionima inuus (Rothschild \& Jordan)}

Material examined: Corrientes. Dpto. San Cosme: $1{ }^{\lambda}$, San Cosme, Wurth col.; Misiones. Dpto. Candelaria: $1 \hat{\partial}$, Loreto, Ogloblin col.; Dpto. Frontera: $2 \widehat{ }$, Piñalito, X-1954, De Carlo \& Viana col.; Dpto. Iguazú: $1 \hat{\jmath}, 1+$, Puerto Iguazú, 22-III-1934, Hayward col.; 14ðิ, XI-1950, Partridge col.; Dpto. San Pedro: $1 \hat{\gamma}$, San Pedro, 1943, Viana col.

Distribution: Corrientes and Misiones.

\section{Callionima nomius (Walker)}

Material examined: Misiones. Dpto. Iguazú:

1ð, XI-1960, Partridge col.

Distribution: Misiones.

\section{Callionima parce (Fabricius)}

Material examined: Misiones. Dpto. Frontera: 3̊̂̉, Piñalito, X-1954, De Carlo \& Viana col.; Dpto. General Manuel Belgrano: $1{ }^{\lambda}$, San Antonio, 1943, Viana col.; Dpto. Iguazú: 1ㅇ, Puerto

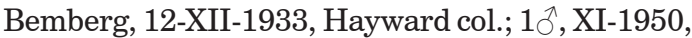
Partridge col.; 1 $\hat{\jmath}$, XI-1954, Partridge col.; 3 $\hat{\circ}$, XI1960, Partridge col.; 2へ, Partridge col. Tucumán. Sin localidad: $1 \delta$, Schreiter col.

Distribution: La Rioja, Misiones, Salta, and Tucumán.

\section{Enyo gorgon (Cramer)}

Material examined: Misiones. Dpto. Apóstoles: 1ㅅ, 1오, Barbeito col.; 1오, Pindapoy, I-1942, Nicolao col.; Dpto. Calendaria: $2+$, Loreto, Ogloblin col.; Dpto. Capital: 10, Posadas, 7-X1933; Dpto. Frontera: $1{ }^{\lambda}$, Piñalito, X-1954, De

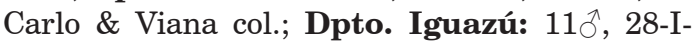

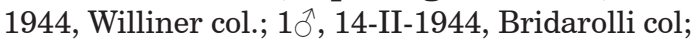
$3{ }^{\lambda}$, XI-1960, Partridge col.

Distribution: Misiones.

\section{Enyo lugubris (Linnaeus)}

Material examined: Buenos Aires. Capital Federal: 1 ${ }^{\lambda}, 28-X I-1947$, Pécora col.; Sin localidad: $1 \hat{\gamma}, 1$, , Nosswitz col.; Chaco. Dpto. San

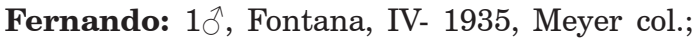
La Rioja. La Rioja: 1 $\widehat{\jmath}$, X-1922, Gómez col.; Misiones. Dpto. Apóstoles: 1앙 Barbeito col.; Dpto. Candelaria: $1 \hat{\jmath}$, Loreto, Ogloblin col.; Dpto. Capital; 1ð̂, Posadas, 14-XII-1933; 1 , Posadas, 19-IX-1933;1 , Posadas; Dpto. Iguazú:

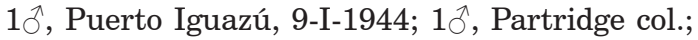
1ð, A. Urugua-í, IX-1954, Orfila col.

Distribution: Buenos Aires, Chaco, Formosa, La Rioja, Misiones, Salta, and Tucumán.

\section{Erinnyis alope (Drury)}

Material examined: Formosa. Sin localidad:

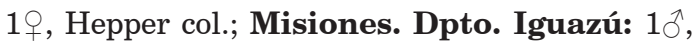

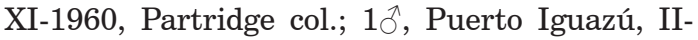
1944, Bridarolli col.; 1ㅇ, Puerto Iguazú, 15-XI1944, Bridarolli col.; 3ð, 1, A. Urugua-í, IX-

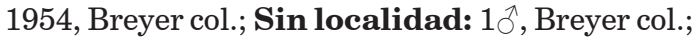

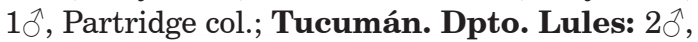
Quebrada de Lules, II-1929; Dpto. Tafí Viejo: 1ภ̂, Tafí Viejo, II-1918; 10̂, Tafí Viejo, III-1918.

Distribution: Buenos Aires, Chaco, Córdoba, Corrientes, Entre Ríos, Formosa, Misiones, Santa Fe, Salta, and Tucumán.

\section{Erinnyis crameri (Schaus)}

Material examined: Chaco. Dpto. San Fernando: 1ㅇ, Fontana, IV-1935, Meyer col.; Misiones. Dpto. Iguazú: 1ð, XII-1933, Hayward col.; 10ึ, Puerto Iguazú, 4-II-1944, Bridarolli col.; 1오 Puerto Iguazú, 11-II-1944, Bridarolli col.; 1 ${ }^{\lambda}$, A. Urugua-í, IX-1954, Orfila col.; 10̂, Cataratas de Iguazú, X-1954, De Carlo

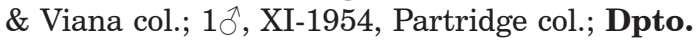
San Pedro: $1 \hat{\gamma}$, San Pedro, 1943, Viana col. Distribution: Chaco, Corrientes, Formosa, La Rioja, Misiones, and Tucumán.

\section{Erinnyis domingonis (Butler)}

Material examined: Chaco. Dpto. San Fernando: 1엉 Fontana, X-1936, Meyer, col.; Misiones. Dpto. Iguazú: $1 \partial^{\lambda}$, Cataratas de Iguazú, X-1954, De Carlo \& Viana col.

Distribution: Chaco, Córdoba, La Rioja, Misiones, Salta, and Tucumán.

\section{Erinnyis ello (Linnaeus)}

Material examined: Buenos Aires. Capital Federal: 1 ${ }^{\lambda}$, II-1927, Orfila col.; 1ㅇ, III-1954, Rivas col.; 2ᄋ, III-1930, Orfila col.; Dpto. 
Saladillo: 1क, Saladillo; 10, 30-III-1904,

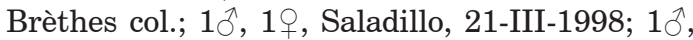
1ㅇ, Saladillo, 27-III-1998; 1ิึ, Verónica F.C.S., III-1938, Daguerre col.; Sin localidad: 1\%, 27-II-1905, Brèthes col.; Chaco. Dpto. San Fernando: 10ิ, Fontana, IV-1935, Meyer col.; Córdoba. Dpto. de Calamuchita: $1 \hat{\jmath}, 1$, El Sauce, III-1972, Viana col.; Sin localidad:

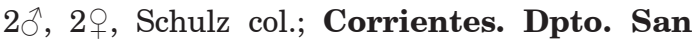
Cosme: 1ㅇ, San Cosme; 1ㅇ, 1935, Wurth col.; Entre Ríos. Dpto: Concordia: $1 \hat{\alpha}$, Estación Experimental de Concordia, XII-1935, Hayward

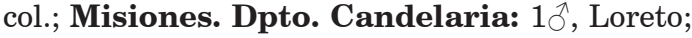

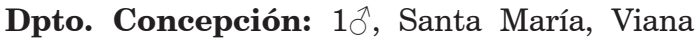
col.; Dpto. Frontera: 1, San Antonio, Viana col.; Dpto. Guarani: 1 $\hat{\jmath}$, El Soberbio, Viana col.; Dpto. Iguazú: 1ภ, 1우, Pto. Bemberg, 1-4XII-1933, Hayward col.; 1 $\hat{O}^{2}, 1$, , Pto. Bemberg, 5-9-XII-1933, Hayward col.; 10, Pto. Bemberg, XII-1933, Hayward col.; 1 ${ }^{\AA}$, XII-1933, Hayward col.; 1ð, Puerto Iguazú, I-1944, Bridarolli col.; 1우, Puerto Iguazú, 14-II-1944, Bridarolli col.;

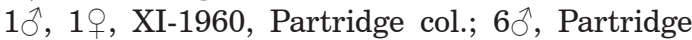

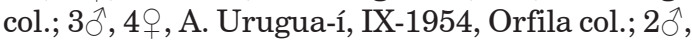
XI-1954, Partridge col.; 6 0 , 1, XI-1960, Viana col.; Dpto. Leandro N. Alem: $1 \jmath^{\hat{n}}$, Leandro N. Alem, 1953, Orfila col.; Dpto. San Javier:

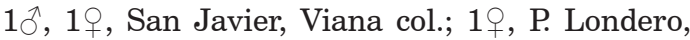
Viana col.; Dpto. San Pedro: 1오 San Pedro, Viana col.; Neuquén. Dpto. Lácar: 10̂, San Martín de los Andes, Parque Nacional Lanín, XII-1951, Schajovskoi col.; Salta. Dpto. Orán: 1우, Urundel, 5-III-1947; Río Caraparí: 1운, I-1945, Bridarolli col.; 1ㅇ, 6-II-1945, Williner col.; San Juan. Sin localidad: 1 ,, $16-\mathrm{X}-1993$; Tucumán. Dpto. Chicligasta: 1 , $1 \hat{\jmath}$, Parque Nacional Campo de Los Alisos, Puesto Santa Rosa, 14-III-2014, Rodríguez-Ramírez col.; 1ㅇ, 3 $\widehat{\jmath}$, Parque Nacional Campo de Los Alisos, Puesto Los Chorizos, 15-16-III-2014, RodríguezRamírez col.; Dpto. Lules: 1ڤึ, 1ㅇ, 27-II-1998; Dpto. Tafí Viejo: 1 $\widehat{\partial}$, Tafí Viejo, XII-1907; 3우, Tafí Viejo, II-1918; 1ð, Tafí Viejo, X-1947, Orfila col.; Dpto. Yerba Buena: $1 \hat{\jmath}$, Reserva Experimental Horco Molle, X-1960, Williner col.; Sin localidad: 1ㅇ, 28-II-1998; 10, 24-V-1998;

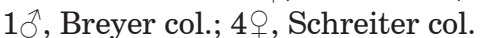

Distribution: Buenos Aires, Catamarca, Chaco, Córdoba, Corrientes, Entre Ríos, Jujuy, La Rioja, Misiones, Neuquén, Salta, San Juan, and Tucumán.

\section{Erinnyis lassauxii (Boisduval)}

Material examined: Buenos Aires. Partido General Alvarado: 1, Miramar, 21-III-1947,
Bachmann col.; Type: 1 q syntype of Dilophonota cercyon Burmeister (see above); 1ㅇ, Gallardo col.; Misiones. Dpto. 25 de Mayo: 11 , Alba Posse, 1947, Viana col.; Tucumán. Dpto. Lules: 1웅, 27-II-1998.

Distribution: Buenos Aires, Córdoba, Chaco, Jujuy, Misiones, Salta, and Tucumán.

\section{Erinnyis obscura (Fabricius)}

Material examined: Buenos Aires. Sin localidad: 1어, 1-II-1901, Zotta col.; Chaco. Dpto. San Fernando: 1엉 Fontana, IV-1935, Meyer col.; 1§, Fontana, X-1936, Meyer col.; Córdoba. Sin localidad: $1 \hat{\jmath}, 1+$, Schulz col.; La Rioja. Sin localidad: $1 \hat{\partial}, 1+$, Giacomelli col.; Misiones. Dpto. Capital: 19 , Posadas, 1-XI1933; Dpto. Iguazú: 1 , , Puerto Bemberg, XII1933, Hayward col.; 1ิ̂̃, Puerto Iguazú, I-1944, Williner col.; 1옹 Puerto Iguazú, 4-II-1944,

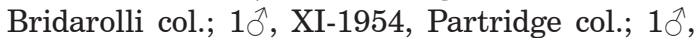
Partridge col.; Dpto. San Javier: $1 \delta$, Puerto Londero, Viana col.; Sin localidad: 1 , X-1928, Breyer col.; Salta. Río Caraparí: 1 3 , 5-I-1945, Williner col.; Tucumán. Dpto. Chicligasta: 1ㅇ, Parque Nacional Campo Los Alisos, Puesto Los Chorizos, 15-16-III-2014, Rodríguez-Ramírez col.; Dpto. Tafí Viejo: 2 , , Tafí Viejo, I-1918; 1 , Manchalá, XII-1922; Sin localidad: $1{ }^{\lambda}$, Breyer col.; $1{ }^{\hat{\prime}}, 2$, , Breyer col.

Distribution: Buenos Aires, Catamarca, Chaco, Córdoba, Corrientes, Entre Ríos, Formosa, Jujuy, La Rioja, Misiones, Salta, Santa Fe, and Tucumán.

\section{Erinnyis oenotrus (Cramer)}

Material examined: Buenos Aires. Typo: 1 syntype of Dilophonota hippothoon Burmeister (see above); Catamarca: 1오 Las Parras, 28-II-1916; Misiones. Dpto. General Manuel Belgrano: 7ో, 1, 1943, Viana col.; Dpto. Guaraní: 1ㅅ, Monteagudo, Viana col.; Dpto. Iguazú: $4 \AA, 3$, A. Urugua-1́, IX-1954, Orfila col.; 3 ${ }^{1}$, Cataratas de Iguazú, X-1954, De Carlo \& Viana col.; 10ิ,

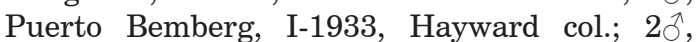
Puerto Bemberg, 4-XII-1933, Hayward col.; 1온 Puerto Bemberg, 5-9-XII-1933, Hayward col.; 1ิ̂, 1, Puerto Bemberg, I-1934, Hayward

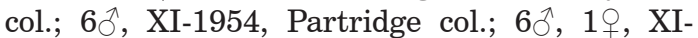
1960, Partridge col.; 22 $\widehat{\text {, }}$ Partridge col.; Dpto. Libertador General San Martín: 10 , Puerto Rico, 1943, Viana col.; Dpto. San Javier: 3 3 , Puerto Londero, Viana col.; San Luis. Dpto. Chacabuco: $1 \hat{\jmath}$, Naschel, XI-1970, Viana col.; Tucumán. Dpto. Lules: $1 \hat{\jmath}, 1$ 우, Quebrada de

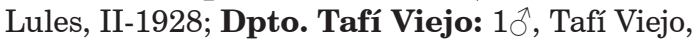


X-1947, Orfila col.

Distribution: Buenos Aires, Catamarca, Córdoba, Jujuy, Misiones, Salta, San Luis, and Tucumán.

\section{Eupyrrhoglossum sagra (Poey)}

Material examined: Misiones. Dpto. Loreto:

1ठ, 1q, Ogloblin col.

Distribution: Buenos Aires, Chaco, and

Misiones.

\section{Hemeroplanes longistriga (Rothschild \& Jordan)}

Material examined: Misiones. Dpto. Iguazú: 1ㅇ, Puerto Iguazú, I-1934, Hayward col.; 1 $\widehat{\jmath}$, X11954, Partridge col.; 1 ${ }^{\dagger}$, X1-1960, Partridge col.; $4 \hat{0}, 1$, , Partridge col.

Distribution: Misiones.

\section{Madoryx bubastus (Cramer)}

Material examined: Misiones.

Candelaria: 1오 Loreto, Ogloblin col.

Distribution: Misiones.

\section{Madoryx oiclus (Cramer)}

Material examined: Misiones. Dpto. Iguazú: 1̊̄, XI-1960, Partridge col.

Distribution: Misiones and Salta.

\section{Nyceryx alophus (Boisduval)}

Material examined: Buenos Aires. Partido de Baradero: 1 $\hat{\jmath}$, Baradero; Partido de Ituzaingó: 19 , Ituzaingó; Sin localidad: 19 , II1918; Misiones. Dpto. Candelaria: 1 우, Loreto, IV-1951; Dpto. Concepción: 1ð̂, Santa María, Viana col.; Dpto. Iguazú: $4 \overbrace{}^{\lambda}, 1$, A. Urugua-í, IX-1954, Orfila col.; 3ð̂, XI-1950, Partridge col.;

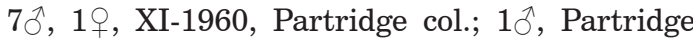
col.; Dpto. San Javier: $1 \hat{\alpha}$, Puerto Londero, 1947, Viana col.

Distribution: Buenos Aires, Chaco, Entre Ríos, and Misiones.

\section{Pachylia darceta (Druce)}

Material examined: Misiones. Dpto. Iguazú: 2へ̂, Puerto Iguazú, I-1934, Hayward col.

Distribution: Misiones.

\section{Pachylia ficus (Linnaeus)}

Material examined: Buenos Aires. Partido La Plata: 1ㅅ, La Plata, Boso col.; Dpto.

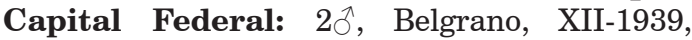
Orfila col.; Misiones. Dpto. Candelaria: $1 \hat{\jmath}$, Loreto, Ogloblin col.; Dpto. General Manuel Belgrano: 1오 San Antonio, 1943, Viana col.; Dpto. Iguazú: 1 $\widehat{\partial}$, XI-1960, Partridge col.;
Tucumán. Dpto. Tafí Viejo: $1 \widehat{\jmath}$, Tafí Viejo, II1918.

Distribution: Buenos Aires, Córdoba, Formosa, Jujuy, Misiones, Salta, and Tucumán.

\section{Pachylia syces (Hübner)}

Material examined: Buenos Aires: 1으, Capital Federal, XI-1927, Orfila col.

Distribution: Buenos Aires, Entre Ríos, Salta, and Misiones.

\section{Pachylioides resumens (Walker)}

Material examined: Buenos Aires. Dpto. Pilar: 1q, Zelaya, X-1936, Pereyra col.; Formosa. Sin localidad: $1 \hat{\sigma}^{\hat{n}}$, 28-III-1916; Misiones. Dpto. Apóstoles: 1우 Dpto. Apóstoles, Apóstoles, Barbeito col.; Dpto. Calendaria: $1 \hat{\jmath}$, Loreto, Ogloblin col.; Dpto. General Manuel Belgrano: 1 ${ }^{\lambda}$, San Antonio, 1943, Viana col.; Dpto. Iguazú: 2へ̂, A. Urugua-í, IX-1954, Orfila col.; 4 ${ }^{\lambda}$, XI-1954, Partridge col.; 4^, 1우, XI-1960, Partridge col.; 2ㅇ, Puerto Bemberg, X-1934, Hayward col.; Sin localidad: $1 \hat{}$, Partridge col.; 1\%, X-1926, Breyer col.;

Distribution: Buenos Aires, Chaco, Córdoba, Corrientes, Entre Ríos, Formosa, Jujuy, La Rioja, Misiones, Salta, and Tucumán.

\section{Perigonia ilus (Boisduval)}

Material examined: Misiones. Dpto. Candelaria: 1 $\hat{\text {, }}$ Loreto, IV-1931; Dpto. Iguazú: 1ㅇ, A. Urugua-í, IX-1954, Orfila col.; 1 $\widehat{\jmath}$, XI-1960, Partridge col.; Dpto. San Pedro: 2今, San Pedro, 1943, Viana col.

Distribution: Misiones.

\section{Perigonia passerina Boisduval}

Material examined: Buenos Aires. Dpto. La Plata: 1ð̂, Isla Martín García, 1-X-1921; Misiones. Dpto. Iguazú: $1 \hat{\alpha}$, Puerto Iguazú, 21-I-1944, Williner col.; 1ð̂, Puerto Iguazú, 28I-1944, Williner col.; Salta. Dpto. General José de San Martín: 1요 Río Caraparí, 13I-1945, Bridarolli col.; 10̂, Río Caraparí, 19-I1945, Williner col.; 1웅 Río Caraparí, 5-II-1945, Williner col.

Distribution: Buenos Aires, Misiones, and Salta.

\section{Phryxus caicus (Cramer)}

Material examined: Buenos Aires. Sin localidad: 1 . .

Distribution: Buenos Aires and Misiones.

Unzela japix discrepans Walker

Material examined: Misiones. Dpto. Iguazú: 
1ㅇ, Iguazú, 13-III-1945, Hayward-WillinerGolbach col.

Distribution: Misiones.

\section{Macroglossinae}

Philampelini

\section{Eumorpha analis (Rothschild \& Jordan)}

Material examined: Buenos Aires. Capital Federal: 1ㅇ, 20-XI-1936; 1ㅇ, Fainbabis col.; 1ิㄱ, 2ㅇ; 1ㅇ, 20-III-1946, Bachmann col.; 1ㅇ, Núñez, 20-II-1939, Rodríguez col.; Partido de Quilmes: 1 ${ }^{\lambda}$, 1q, Bernal, II-1936, Pérez \& Morean col.; Partido de San Miguel: $1+$, Bella Vista, III-1991, Gallardo col.; Sin localidad: 1ิ , 27-IV-1926; 10̂, 31-X-1904, Brèthes col.; Catamarca. Sin localidad: 1 , , Breyer col.; Córdoba. Capital: 1을 Barrio Alta Córdoba, 2-III-1931, Williner col.; Dpto. Calamuchita:

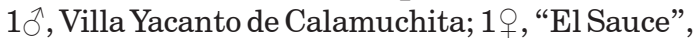
III-1972, Viana col.; Sin localidad: 10, 1q, Schulz col.; Misiones. Dpto. Candelaria: 1 , Loreto, Ogloblin col.; Dpto. General Manuel Belgrano: $3 \hat{\jmath}, 1$, , San Antonio, Viana col.; $1 \hat{\jmath}$, San Antonio, 1943, Viana col.; Dpto. Iguazú:

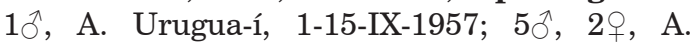
Urugua-1́, IX-1954, Orfila col.; 5 ${ }^{\hat{\prime}}$, Partridge col.; 4へ, XI-1950, Partridge col.; 15 , 2 , XI-1954, Partridge col.; Dpto. Libertador General San Martín: 1ð̂, Puerto Rico, 1943, Viana col.; San Pedro: $1 \hat{\jmath}$, San Pedro, 1943, Viana col.; Dpto. 25 de Mayo: $2 \hat{\jmath}$, Alba Posse, Viana col.; Salta. Dpto. General José de San Martín: $1 \hat{\circ}$, Río Caraparí, 6-II-1945, Williner col.; Tucumán. Dpto. Yerba Buena: $1 \hat{\jmath}$, Reserva Experimental Horco Molle, X-1960, Williner col.; 2ð̂̀, Manchalá, I-1922.

Distribution: Buenos Aires, Catamarca, Córdoba, Formosa, Jujuy, La Rioja, Misiones, Salta, San Luis, and Tucumán.

\section{Eumorpha anchemolus (Cramer) \\ Material examined: Misiones. Dpto.} Calendaria: 1, Loreto, Ogloblin col.; Dpto. Iguazú: 1ð̂, Puerto Bemberg, XII-1931, Pallotto col.

Distribution: Catamarca and Misiones.

\section{Eumorpha fasciatus (Sulzer)}

Material examined: Buenos Aires. Sin localidad: 10, III-1952, Orfila col.; 1우, 21-I-1902; Dpto. Tigre: $1 \hat{\gamma}$, 1우, Tigre, II-1932, Bourquin col.; Córdoba. Sin localidad: $1 \hat{\jmath}, 1$, Schulz col.; Misiones. Dpto. Apóstoles: 19, Apóstoles, 1936, Orfila col.; Dpto. Iguazú: 1, XI-1960,
Partridge col.; Salta. Dpto. Orán: 10, Urundel, 28-II-1947; Santa Fe. Dpto. Rosario: 1 $\sigma^{\widehat{ }}$, Rosario, Stévenin col.; Tucumán. Dpto. Tafí Viejo: 1 , Tafí Viejo.

Distribution: Buenos Aires, Córdoba, Corrientes, Chaco, Formosa, Misiones, Salta, Santa Fe, and Tucumán.

\section{Eumorpha labruscae (Linnaeus)}

Material examined: Córdoba. Dpto. Colón: 1ภ, Unquillo, 15-V-1938, Buffo col.; Sin localidad: $1 \hat{\jmath}$, Schulz col.; $1 \hat{O}^{\hat{2}}$ II-1948, Giai col.; Corrientes. Sin localidad: 19,1935 , Wurth col.; Jujuy: 1ㅇ, Ledesma, 28-XI-1978, Williner col.; Misiones. Dpto. Apóstoles: 1q, Apóstoles, 1936, Orfila col.; Dpto. Iguazú: 1ठ, XI-1960, Partridge col.; 2 ${ }^{2}$, XI-1954, Partridge col.; Santa Fe. Dpto. Rosario: 2へ, Rosario, Stévenin col.; Tucumán. Manchalá: 1ð̂, I-1920; 1ठ̂, I-1922, 2 , II-1922.

Distribution: Buenos Aires, Córdoba, Corrientes, Entre Ríos, Jujuy, Mendoza, Misiones, Río Negro, Salta, Santa Fe, and Tucumán.

\section{Eumorpha neuburgeri (Rothschild \& Jordan)}

Material examined: Tucumán. Dpto.

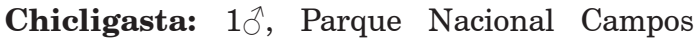
Los Alisos, Puesto Santa Rosa, 14-III-2014,

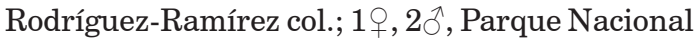
Campos Los Alisos, Puesto Los Chorizos, 15-16III-2014, Rodríguez-Ramírez col.; Dpto. Lules: 4우, Quebrada de Lules, II-1928; Sin localidad: 2 ㅇ, Orfila col.; $3{ }^{\lambda}$, Scheiter col.; 1우, Breyer col.; Distribution: Catamarca, Jujuy, La Rioja, Salta, and Tucumán.

\section{Eumorpha vitis (Linnaeus)}

Material examined: Entre Ríos. Dpto. Concordia: 1오 San Antonio de Padua de la Concordia, Estación Experimental de Concordia; Tucumán: 1, Manchalá, I-1922; 10̄, Manchalá, II-1922.

Distribution: Buenos Aires, Córdoba, Corrientes, Entre Rios, Formosa, Jujuy, La Rioja, Salta, Santa Fe, and Tucumán.

\section{Macroglossinae Macroglossini}

\section{Hyles annei (Guérin-Meneville)}

Material examined: Mendoza. Sin localidad:

1 으, Reed col.

Distribution: Mendoza and San Juan. 
Hyles euphorbiarum (Guérin-Méneville \& Percheron)

Material examined: Buenos Aires. Sin localidad: 1, II-1939, Orfila col.; Chubut: 1ㅇ, Península Valdés, 3-III-1970, Daciuk col.; Dpto. Biedma: 1ㅇ, Puerto Madryn, III-1937, Pascheto col.; Córdoba. Dpto. de Calamuchita: 1 우, El Sauce, III-1972, Viana col.; 10̂, Santa Rosa

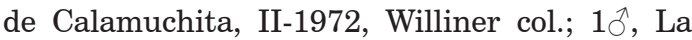
Tablada; Sin localidad: $2 \hat{\jmath}, 1$, Schulz col.; La Pampa. Sin localidad: 2 , Breyer col.; La Rioja. Dpto. Independencia: 1 , Patquía; Sin localidad: $4 \hat{\jmath}, 3+$, Giacomelli col.; Mendoza. Dpto. La Paz: 1q, Desaguadero, XII-1972, Williner col.; Misiones. Dpto. Iguazú: 1우 A. Urugua-í, IX-1954, Orfila col.; Neuquén. San Martín de Los Andes: $3 \hat{\jmath}, 1$, , Schajovskoi col.;

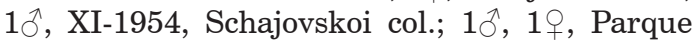
Nacional Lanín, XI-1949, Schajovskoi col.; 1우, Parque Nacional Lanín, XII-1951, Schajovskoi col.; Salta. Dpto. General José de San Martín: 1우 Río Caraparí, 19-I-1945, Williner col.; San Juan. Dpto. Valle Fértil: 2 , II1972, Viana col.; San Luis. Dpto. Juan Martín de Pueyrredón: $1 \hat{\jmath}, 1$ 옹 San Gerónimo, 23-XI1971, Williner col.; 19, San Gerónimo, XI-1972, Williner col.; 2ภ, 3오, San Gerónimo, XII-1972, Williner col.; 1ð, 1, San Gerónimo, XII-1972, Viana col.; Tucumán. Dpto. Lules: 1ڤ̂, Lules,

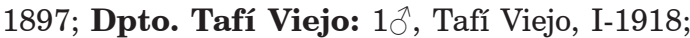
Sin localidad: 1 ,, $24-\mathrm{V}-1998$.

Distribution: Buenos Aires, Catamarca, Chubut, Córdoba, Corrientes, Jujuy, La Pampa, La Rioja, Mendoza, Misiones, Neuquén, Salta, San Juan, San Luis, Tierra de Fuego, and Tucumán.

\section{Hyles lineata (Fabricius)}

Material examined: Buenos Aires. Dpto. Bahía Blanca: 1ô, Bahía Blanca, 14-IV-1927, Renard col.; Catamarca. Dpto. Andalgalá:

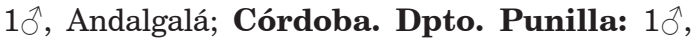
Capilla del Monte; $3 \hat{\jmath}$, La Tablada; Sin localidad: 1 , , Giacomelli col.; 1우, Schulz col.; Dpto. Calamuchita: 10, Villa General Belgrano, 27-X-1971, Williner col.; 1ðै, Santa Rosa de Calamuchita, Viana col.; 1, Los Reartes, 1972, Viana col.; 3ㅇ, El Sauce, III-1972, Viana col.; Dpto. Santa María: 1오, Alta Gracia, II1954; Entre Ríos. Dpto. Uruguay: $1 \hat{\jmath}$, Las Moscas, 1940, Orfila col.; Jujuy. Sin localidad: 1今, XI-XII-1925, Pozzi \& Zotta col.; San Luis. Dpto. Juan Martín de Pueyrredón: 19 , San Gerónimo, XII-1972, Viana col.; Tucumán. Dpto: Tafí Viejo: $1 \hat{\jmath}$, Tafí Viejo, I-1918; $2 \hat{\jmath}$, Tafí Viejo, II-1918; 1ㅇ, Tafí Viejo, XII-1917.
Distribution: Buenos Aires, Catamarca, Córdoba, Entre Ríos, Jujuy, La Rioja, Salta, San Luis, Santa Fe, and Tucumán.

\section{Xylophanes ceratomioides Grote \&} Robinson

Material examined: Salta. Manuel Elordi: $1{ }^{\jmath}$. Distribution: Jujuy, Misiones, and Salta.

\section{Xylophanes chiron (Drury)}

Material examined: Misiones. Dpto. Frontera: 1ð, 19, Piñalito, X-1954, De Carlo \& Viana

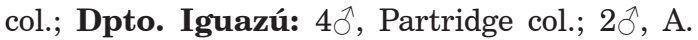
Urugua-í, IX-1954, Orfila col.; 2 + , Pto. Bemberg, 4-XII-1933, Hayward col.; 10ิ, XI-1960, Partridge

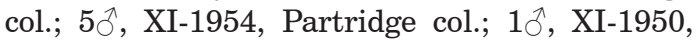
Partridge col.

Distribution: Buenos Aires, Misiones, Salta, and Tucumán.

\section{Xylophanes isaon (Boisduval)}

Material examined: Misiones. Dpto. Iguazú: 1ठ, XII-1931, Pallotto col.

Distribution: Misiones.

\section{Xylophanes loelia (Druce)}

Material examined: Misiones. Dpto. Iguazú: 2ð, 2ᄋ, Pto. Bemberg, 1-XII-1933, Hayward col.; 1ㅇ, X-1928, Breyer col.; Sin localidad: $1{ }^{\widehat{T}}$, Nosswitz col.

Distribution: Misiones.

\section{Xylophanes marginalis Clark}

Material examined: Misiones. Dpto. Iguazú: 1ㅇ, Pto. Iguazú, 22-X-1947, Ramos col.

Distribution: Misiones.

\section{Xylophanes pluto (Fabricius)}

Material examined: Misiones. Dpto. Iguazú: 5^, 1ㅇ, Pto. Bemberg, 1-IV-1934, Hayward col.; Tucumán. Dpto. Lules: 2へ, Quebrada de Lules, II-1928; Dpto. Tafí Viejo: 2 $\widehat{\jmath}, 1+$, Tafí Viejo, I-1918.

Distribution: Corrientes, Jujuy, Misiones, Salta, and Tucumán.

\section{Xylophanes porcus (Hübner)}

Material examined: Misiones. Dpto. Iguazú:

10, XI-1945, Duret col.

Distribution: Misiones.

\section{Xylophanes schreiteri Clark}

Material examined: Tucumán. Dpto. Lules: 2今., Quebrada de Lules, II-1928; Dpto. Tafí Viejo: $1 \widehat{\partial}$, Tafí Viejo, I-1918; $1 \widehat{\partial}$, Tafí Viejo, II- 
1918; 2ð, Schreiter col.

Distribution: Córdoba, Jujuy, and Tucumán.

\section{Xylophanes tersa (Linnaeus)}

Material examined: Buenos Aires. Capital Federal: 1 $\hat{}$, 20-XII-1953, Orfila col.; Partido La Matanza: $1 \hat{\gamma}, 1$, , La Tablada, Giacomelli col.; 7ðे, 3ㅇ, La Tablada; Partido San Isidro: 10̂, Martínez, 10-IV-1928, Gómez col.; Sin localidad: 10ิ, 31-I-1919, Boneo col.; 10, 8-III1906, Brèthes col.; 10̂, 1935; Chaco. Dpto. San

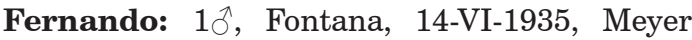
col.; Córdoba. Capital Córdoba: $1 \hat{\alpha}$, Barrio Alta Córdoba, 22-III-1980, Williner col.; Dpto. Calamuchita: $3 \hat{\circ}, 3$ ㅇ, Dpto. Calamuchita, El Sauce, III-1972, Viana col.; Dpto. Ischilín: 1웅 Deán Funes; Dpto. Punilla: 2 $\overbrace{}^{\Uparrow}$, Tanti, II-1950, Viana col.; 1 $\widehat{\jmath}, 3$, , Cosquín, Giacomelli col.; $2 \hat{\jmath}$, 1ㅇ, Cosquín; Dpto. San Javier: 4수, 1 , La Paz, 1-15-I-1929, Bruch col.; Sin localidad: 1 , Schulz col.; 10̄, Giacomelli col.; Corrientes. Sin localidad: 1 ㅇ, 1935, Wurth; Entre Ríos. Dpto. Colón: 19, Obrador, 24-X-1976, Williner col.; Jujuy. Dpto. Doctor Manuel Belgrano: 1ㅇ, San Salvador de Jujuy, II-1959; La Rioja. Sin localidad: $1 \hat{\jmath}$, Giacomelli col.; Misiones. Dpto. Apóstoles: 1 $\delta$, VII-1936, Castillo col.; Dpto. Candelaria: 1으, Loreto, Ogloblin col.; Dpto. Capital: $2 \hat{\jmath}$, Posadas, 10-30-XI-1933, Hayward col.; 10ิ, Posadas, 21-IX-1933; 20, Posadas; Dpto. Frontera: 1올 Piñalito, X-1954, De Carlo \& Viana col.; Dpto. Iguazú: 1웅 X-1954, Partridge col.; 2 , , Cataratas de Iguazú, X-1954, De Carlo \& Viana col.; 3 $\widehat{\text {, }}$, Partridge col.; 2へ̂, Puerto Iguazú, 15-II-1944, Bridarolli col.; $3 \hat{\text { A }, 1}$ ㅇ, Puerto Bemberg, 5-9-XII-1933, Hayward col.; Dpto. San Javier: 1 , , Puerto Londero, XI1947, Viana col.; Salta. Ciudad Salta: $1 \hat{\jmath}$, Barrio San Benito, IV-1975, Martínez col.; San Luis. Dpto. Coronel Pringles: 1요 Peñón Colorado, La Carolina, V-1931; 2§̂, Dpto. Juan Martín de Pueyrredón, San Gerónimo, XI-1972, Williner col.; Dpto. Juan Martín de Pueyrredón: 2へ, San Gerónimo, 3-II-1972, Williner col.; Santa Fe.: 1울 Ciudad de El Piquete, 21-I-1929, Muhn col.; Tucumán. Dpto. Tafí Viejo: $2 \hat{\diamond}, 2+$, Tafí Viejo, II-1918; Sin localidad: $1 \hat{\partial}, 1$, , Breyer col.

Distribution: Buenos Aires, Catamarca, Chaco, Córdoba, Corrientes, Entre Ríos, Formosa, Jujuy, La Rioja, Misiones, Salta, San Luis, Santa Fe and Tucumán.

\section{Xylophanes thyelia (Linnaeus)}

Material examined: Misiones. Dpto. Iguazú:
1วิ, XII-1931, Pallotto col.

Distribution: Misiones.

\section{Xylophanes titana (Druce)}

Material examined: Misiones. Dpto. Iguazú: 3ิे, Puerto Bemberg, 16-XII-1933, Hayward col.; $1 \delta$, A. Urugua-í, IX-1954, Orfila col.

Distribution: Jujuy, Misiones, and Salta.

\section{ACKNOWLEDGMENTS}

I would like to thank Ezequiel Nuñez-Bustos for his help providing a revision of the ordered and classified specimens, which confirms the identity of several species, as well as for his advice and suggestions as to the continuous study of this family. Likewise, I would like to thank Dr. Arturo Roig-Alsina for his broad and excellent help both in the edition of this publication and in the contribution and opinion within the frame of these results, especially in relation to the types. Furthermore, I am very grateful to Ángel Fusaro who contributed to the results with his excellent pictures giving further interest to these beautiful nocturnal butterflies, which in turn received help for their edition thank to Pablo Mullieri and Sofía Olea. Finally, I would like to thank Gretel Caminos for her help in the review of grammar of the entire text of the publication.

\section{BIBLIOGRAPHY}

Beccacece, H.M., A.I. Zapata, N.A. Villafañe, A. Zarco, M.P. Cherini \& M.E. Drewniak. 2011. Riqueza de esfíngidos (Lepidoptera: Sphingidae) en el Bosque Serrano de la Reserva Hídrica Natural "Parque La Quebrada" y sus alrededores (Córdoba, Argentina). Rev. Soc. Entomol. Argent. 70(1-2): 137-140

Berg, C. 1883. Miscelanea Lepidopterologica. Contribuciones al estudio de la Fauna Argentina y países limítrofes. An. Soc. Cient. Argent. XV: 151152.

Berg, C. 1885. Quindecim. Lepidoptera Nova. Faunae Republicae Argentinae et Uruguayensis. . An. Soc. Cient. Argent. XIX: 266-267.

Burmeister, H. 1878. Lépidoptères (Parte 1). En: Description physique de la République Argentine, volume 5, Coni, Buenos Aires, Savy, Paris, Anton, Halle, 355 pp.

Burmeister, H. 1879. Lépidoptères (Parte 2). Atlas. En: Description physique de la République Argentine, volume 5, Coni, Buenos Aires, Savy, Paris, Anton, Halle, 64 pp + 24 lám.

Giacomelli, E. 1912. Sobre una nueva especie de Protoparce. (Sphingidae). An. Soc. Cient. Argent. LXXIV: 55-57.

Haber, W.A. \& Frankie, G.W. 1989. A tropical hawkmoth community: Costa Rican dry forest Sphingidae. Biotropica 21(2): 155-172. 
Kitching, I.J. \& J.M. Cadiou. 2000. Hawkmoths of the World: An Annotated and Illustrated Revisionary Checklist (Lepidoptera: Sphingidae). Cornell University Press, Ithaca \& London, $227 \mathrm{pp}$.

Köhler, P. 1924. Fauna Argentina. Lepidoptera e collectione Alberto Breyer. II. Teil. Heterocera. Systematischer Katalog und Studien, Berichtigungen, Neubeschreibungen. Zeit. Wiss. Insektenbiol. 19(suppl): 1-28.

Mattoni, R., F. Penco. 2012. Big Moths of Buenos Aires and Southern Uruguay. The Lepidoptera Research Foundation, Beverly Hills, California, 35 pp.

Moré, M., I.J. Kitching, \& A.A. Cocucci. 2005. Sphingidae: Esfingidos de Argentina. Hawkmoths of Argentina: L. O. L. A., Buenos Aires, 166 pp.

Nuñez-Bustos, E. 2008. Las especies de Sphingidae de la Reserva Privada Yacutinga, provincia de Misiones, Argentina (Lepidoptera: Sphingidae). SHILAP Revta. Lepid. 36(142): 219-226.

Nuñez-Bustos, E. 2009. Sphingidae de la zona de Aguas Blancas, provincia de Salta (Argentina), con comentarios sobre nuevos registros provinciales (Lepidoptera: Sphingidae). SHILAP Revta. Lepid. 37(147): 363-369.

Nuñez-Bustos, E. \& J. Rodríguez-Ramírez. 2014. Dos nuevos registros de Macroglossinae (Lepidoptera: Sphingidae) de la Argentina. Rev. Soc. Entomol. Argent. 73(1-2): 71-73.

Oehlke, B. 2014. Sphingidae of the Americas. Disponible en: http:// www.silkmoths.bizland.com/ danjansphinx.htm (Agosto de 2014).

Orfila, R. 1933. Estudios de Lepidopterología Argentina III. Catálogo sistemático de los Sphingidae (Lep.). Revta. Soc. Entomol. Argent. 5(2-3): 189-206.

Pastrana, J. A. 2004. Los lepidópteros argentinos. Sus plantas hospedadoras y otros sustratos alimenticios. Sociedad Entomológica Argentina, Buenos Aires, 334 pp.

Rothschild, L. W. \& K. Jordan. 1903. A revision of the lepidopterous family Sphingidae. Novitates zoologicae. 9(Supl.): 1-972.

Schreiter, R. 1926. Sphingidae. Estudio sobre las especies tucumanas de esta familia. Bol. Mus. Cienc. Nat. Tucumán. 1(9): 1-24.

Schreiber, H. 1978. Dispersal centres of Sphingidae (Lepidoptera) in the Neotropical Region. Biogeographica. Junk B.V., The Hague: 195 pp.

Valverde, L. 1999. Sphingidae del Parque Nacional Calilegua, Jujuy, Argentina (Insecta: Lepidoptera). SHILAP Revta. Lepid. 27(106): 215-223. 